\title{
Chromospheric Prolateness: Solar Cycle Variations
}

\author{
J. Vilinga ${ }^{1}$, S. Koutchmy ${ }^{1}$, Fr. Auchere ${ }^{2}$, Fr. Baudin ${ }^{2}$, B. Filippov ${ }^{3}$ \\ and J-C. Noens ${ }^{4}$ \\ ${ }^{1}$ Institut d'Astrophysique de Paris CNRS and P. \& M. Curie University and University A. \\ Neto in Luanda-Angola \\ email: vilinga@iap.fr koutchmy@iap.fr \\ ${ }^{2}$ Institut d'Astrophysique Spatiale, Orsay France \\ ${ }^{3}$ IZMIRAN, Troitsk, Moscow Region, Russia \\ ${ }^{4}$ OMP and Pic du Midi Observatory, France
}

\begin{abstract}
The solar prolateness (also known as Ovalisation, a french origin name) of the extended dynamical chromosphere is established from measurements performed above $2 \mathrm{Mm}$ heights during the years of solar minimum, using the $\mathrm{H} \alpha$, Ca II K and HeII 304 line emissions from both ground-based and space-based observations. Coronal X-EUV emissions usually penetrate deep enough into the chromosphere to completely mask this effect on transition region lines and produce the so-called coronal hole effect. However, cool lines like $\mathrm{H} \alpha$ and Ca II lines, do NOT show this Coronal Hole $(\mathrm{CH})$ effect. Coronal lines and HeI (D3; $1083 \mathrm{~nm})$ do show $\mathrm{CHs}$ but do not show the prolateness effect. We first briefly review different methods which can potentially be used to measure the prolateness. Further we note the similarity of the geometric behaviour of the prolateness and its variation along the solar cycle compared to the behaviour of the fast solar wind. It suggests the same origin possibly related to the emergence of the small scale network and internetwork magnetic field towards the corona and small scale magnetic reconnections. A simple geometric model was proposed to explain the effect of the prolateness of the solar chromosphere by considering that the specific dynamical part of the solar atmosphere above the $2 \mathrm{Mm}$ level, being a mixture of up and down moving jets of chromospheric matter with the coronal plasma between them, is responsible for the solar prolateness (Filippov and Koutchmy, 2000). We however note that polar regions are also showing different types of activity in the low corona, including small prominence eruptions seen e.g. in $\mathrm{H} \alpha$ and linear jets seen in SXR and EUV as well as in W-L (eclipses). Some kind of dynamical dissipation of the newly emerged magnetic field is needed. More systematic measurements should be done to build a more complete, possibly 3D, picture to explain the extended in the horizontal direction lifting effect of a large part of the polar chromosphere.
\end{abstract}

Keywords. Sun: chromosphere, solar atmosphere, magnetism

\section{Introduction}

Precise measurements of the constant photospheric solar radius in polar and equatorial directions showed a very small difference, of order of 10 milli-arcsec (e.g. Rozelot and Rosch, 1996), making the Sun oblate. This is roughly the value anticipated from the "flattening" effect due to the bulk rotation of the Sun. We discuss here the opposite case of the prolate chromosphere e.g. the extended along the polar axis solar chromosphere that we also call ovalisation. From historic visual and later, photographic $\mathrm{H} \alpha$ observations, it was evident that the polar chromosphere looks more extended than the low latitude chromosphere, at least at solar minimum of activity (Secchi, 1877; Roberts, 
1946, Fracastoro, 1948). However, at ground-based, the differential refraction effects due to the Earth atmosphere should be considered when looking at the whole shape of the chromosphere, see Figure 1.

Johannesson and Zirin (1996) using photometric quality data measured the relative extension of the chromosphere with respect to the photosphere, at $\mathrm{H} \alpha$ center, to reach typically 4.3-4.4 $\mathrm{Mm}$ at the equator and just below $6 \mathrm{Mm}$ at the solar poles, with a true local scatter of $0.5 \mathrm{Mm}$. This is in agreement with the values reported in the literature, see e.g. the Dunn's (1965) thesis, although this extension is still a matter of debate and, moreover, is not taken into account in the modelling of the transition region. Auchere et al. (1998) performed a more statistical comparative analysis of the shape of the corona using several coronal lines and of the chromosphere, using the He II 304 observations. In addition, they described strictly simultaneous $\mathrm{H} \alpha$ ground-based observations and space-based observations with SOHO EIT, see also Koutchmy et al. (1998), Georgakilas, Koutchmy, and Alissandrakis (1999). The solar chromosphere was found to be prolate to an extent of 3 ". $0 \pm 1$ " over a solar diameter in $\mathrm{H} \alpha$ full width line and up to $\sim 12$ " for He II emission, without removing effects due to the weaker radial gradients known to exist above CHs. Huber et al. 1974 were the first to describe this $\mathrm{CH}$ effect in the low corona. The chromospheric effect was independently obtained for the EIT data alone by Zhang et al. (1998). At the time of solar minimum, the limb seen in EIT coronal images is also higher at the poles than at the equator by $1.3 \pm$ $0.65 \mathrm{Mm}$, but this effect is possibly affected by the presence of polar coronal holes. Moreover, the transition region (TR) outer limb seen in He II images is considerably higher than the limb measured in "absorption" on coronal images, see Auchère et al. 1998. The height difference is $3.1 \pm 1.2 \mathrm{Mm}$ at the equator and $6.6 \pm 1.2 \mathrm{Mm}$ at the poles. After considering effects due to the polar coronal holes, the prolateness has been confirmed using new measurements obtained from an observing run at the SP/VTT on August 1998 (Koutchmy et al., 1998). These results were first discussed in Filippov and Koutchmy, 2000.

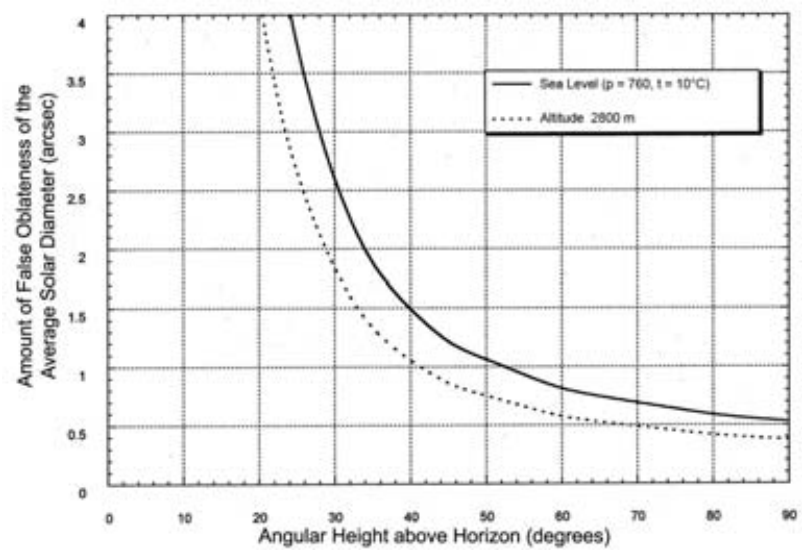

Figure 1. False oblateness due to the Earth atmospheric differential refraction effect computed for both a sea level site and a mountain site of $2800 \mathrm{~m}$ altitude like the Sacramento Peak or the Pic du Midi Observatories. At low altitude sites and low solar elevations this effect is considerably larger than the chromospheric prolateness effect. 


\section{Observations}

One of the problem occurring when precise measurements of the shape of the solar disk are attempted is due to the distortion effects. Spectro-heliograms, for example, cannot be used because the large instrumental distortions introduced when constructing the whole image from slices and non linear effects introduced during the scanning. In addition distortion is usually not reproducible. Even the accurately reconstructed whole disk images recently produced from space with SUMER/SoHO are not good enough. Provided the instrumental distortion is minimised by using properly collimated systems, ground-based filtergrams can be used. Then, the Earth atmospheric distortion should be taken into account. We studied this effect produced by the Earth atmospheric induced differential refraction effect, see e.g. Fig. 1, on the apparent shape of the solar disk. The model can then be used to introduce corrections when measuring the ratio of diameters pole/equator but a more sophisticated algorithm has to be developed to analyse the whole limb profile. It is much easier to measure just the thickness of a part of the chromosphere, around the pole and around the equator, because the correction for differential refraction is then neglected compared to the value of the "local" prolateness.

Accordingly, the prolateness can be evaluated thanks to relative measurements of the thickness of the extended dynamical chromosphere, going well above the $2.1 \mathrm{Mm}$ heights given by the theoretical hydrostatic VAL model atmosphere (Vernazza et al. 1981) for the top of the chromosphere. We performed these measurements using different methods and found the "spectroscopic" method particularly useful. In this method, many spectra are taken along a strictly radial direction, in the region of a low excitation lines, with optimum exposure times to get a good signal to noise ratio on emissions recorded outside the limb (in addition, the scattered light is properly subtracted), without overexposing the disk parts seen in the neighboring continuum. In order to provide relevant results, it is necessary to average the spectra, both in space and in time. We used averaged spectra after processing typically 160 spectra taken near each reference positions (2 polar positions around the North and the South poles, and 2 equator positions, around the positions of the East and of the West limbs). It takes typically $10 \mathrm{~min}$ of time for each position, see Fig. 3. Measurements of the chromospheric thickness are taken with respect to the precise position of the photospheric limbs as measured using the continuum parts of spectra. The limb position is deduced from the position of the center of gravity position of the curves obtained after deriving in space the intensity variations of the extreme limbs curves. The position of the outer "limbs" of the chromosphere are evaluated using the position of the inflection point which is rather close to the position of half width intensities, after subtracting the intensity of the nearby continuum from the intensity of the considered chromospheric emission in order to remove the scattered light. In table 1 we present the results coming from our solar minimum measurements, see also, Filippov and Koutchmy, 2000.

Measurements were later done during the solar maximum using both the filtergrams (with the Themis telescope at Tenerife in 2000) and the spectroscopic method (at NSO/ Sacramento Peak with the HSG of the Dunn's telescope). We could not measure any definite prolateness; sometimes the equatorial chromosphere appears even more extended than the polar one. Average spectra over 160 arcsec around the positions of the poles and of the equators for the spectral region near $\mathrm{H} \alpha$ line using a strictly radial slit of the HSG of the Dunn's solar telescope of NSO/SPO were also used thanks to a run performed in 2000. 160 spectra are obtained at each position using a 1 sec exposure time. No prolateness effect was measured, see Vilinga and Koutchmy, 2005. 

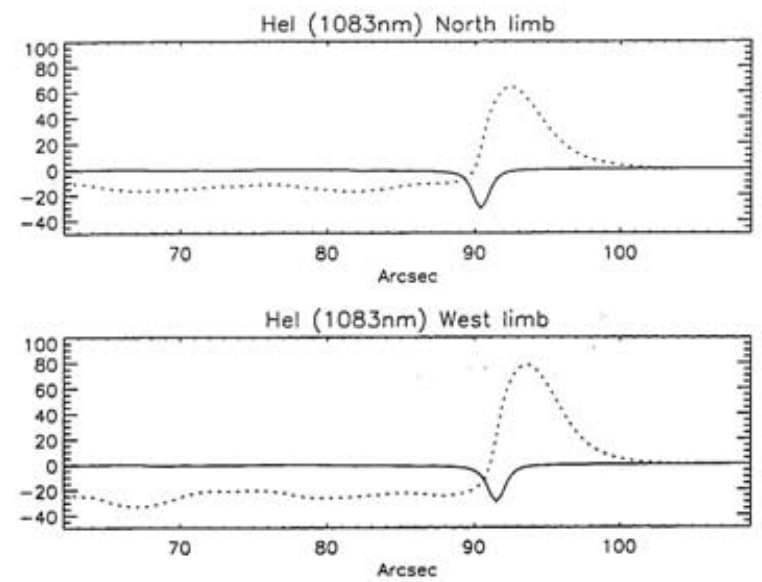

Coll $\mathrm{K}$ North limb

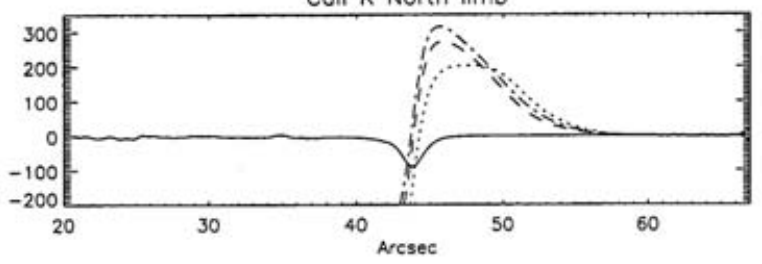

Coll $K$ West limb

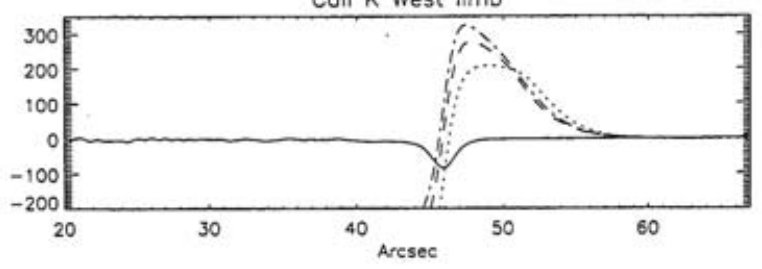

Figure 2. Intensity variations of the chromospheric (dotted and dashed lines) and of the photospheric (derivative profiles in full line) limbs simultaneously measured in 1998 to evaluate the extension of the chromosphere at different positions around the limbs. Line profiles taken over 160 arcsec around each polar and equatorial positions are averaged to deduce these intensities. Curves corresponding to the HeI $1083 \mathrm{~nm}$ (no prolateness but a $\mathrm{CH}$ effect) and to the CaII K line emissions (well pronounced prolateness effect) are shown.

Table 1. Heights of the average limb positions taken above the level $\tau_{5}=1$ as observed in 1998 using different chromospheric spectral lines.

\begin{tabular}{|c|c|c|c|c|c|}
\hline Element & Line & $\begin{array}{l}\text { Height of the } \\
\text { average limb } \\
\text { at equator } \\
(\mathrm{Mm})\end{array}$ & $\begin{array}{l}\text { Height of the } \\
\text { average limb } \\
\text { at poles } \\
(\mathrm{Mm})\end{array}$ & $\begin{array}{l}\text { Prolateness } \\
\text { over a solar } \\
\text { radius } \\
(\mathrm{Mm})\end{array}$ & $\begin{array}{l}\text { Temperature } \\
\text { of line } \\
\text { formation }(\mathrm{K}) \text {; } \\
\text { (from line } \\
\text { width) }\end{array}$ \\
\hline $\mathrm{H}$ & $\mathrm{H} \alpha$ & & & $1.1 \pm 0.35$ & 8000 \\
\hline Ca II & K2 & 2.6 & & & 6500 \\
\hline Ca II & K3 & $3.35 \pm 0.15$ & $3.8 \pm 0.15$ & $0.45 \pm 0.15$ & 6500 \\
\hline He II & $30.4 \mathrm{~nm}$ & $5 \pm 0.5$ & $6.5 \pm$ & $1.5 \pm 5$ & $(50000)$ \\
\hline \multirow{2}{*}{ He I } & $1083 \mathrm{~nm}$ & $1.9 \pm 0.3$ & $1.9 \pm 0.3$ & $0 \pm 0.3$ & $(8000)$ \\
\hline & $\mathrm{D}_{3}$ & $1.9 \pm 0.3$ & $1.9 \pm 0.3$ & $0 \pm 0.3$ & $(8000)$ \\
\hline
\end{tabular}


In space, there is no Earth atmospheric induced distorsion effects. The chromospheric prolateness can then be measured on full disk filtergrams like what is provided by the EIT experiment of SoHO, which, in addition, are almost free of significant instrumental distortion effect. Coronal EIT filtergrams are not suitable to precisely show the chromosphere, although some absorption effect seen near the limb on coronal filtergrams can be attributed to the thick chromosphere. Indeed, some plasma filling the space between the corona and the chromosphere at transition region (TR) temperatures produces this absorption, see Daw et al. 1995. Accordingly, the shape of the limb is completely dominated by effect at high TR temperature, including the effect of coronal holes, see Huber et al. 1974 and Auchere et al. 1998. HeII 304 resonance line emission filtergrams are available to permit a direct evaluation of the shape of the HeII chromosphere. Spaceborne observations of the shape of the limb from full disk images were done using the EIT/SoHO experiment. They mainly show the effect of polar $\mathrm{CHs}$ on the shape of the limbs computed using coronal line emissions. However, the limb positions observed using the HeII 304 line do show the prolateness effect superposed to the rather large effect due to the polar CHs. We show on Fig. 3 a synoptic view of both the limb positions and limb intensities measured in the HeII line by Frederic Auchére during the Years 1996 to 2000 to mainly show the disappearance of the $\mathrm{CH}$ effect presumably corraleted with the disappearance of the prolateness effect seen at groundbased. It is also illustrating how SoHO space-borne observations are superior in quality compared to ground-based data to show a continuous global variation during Years.
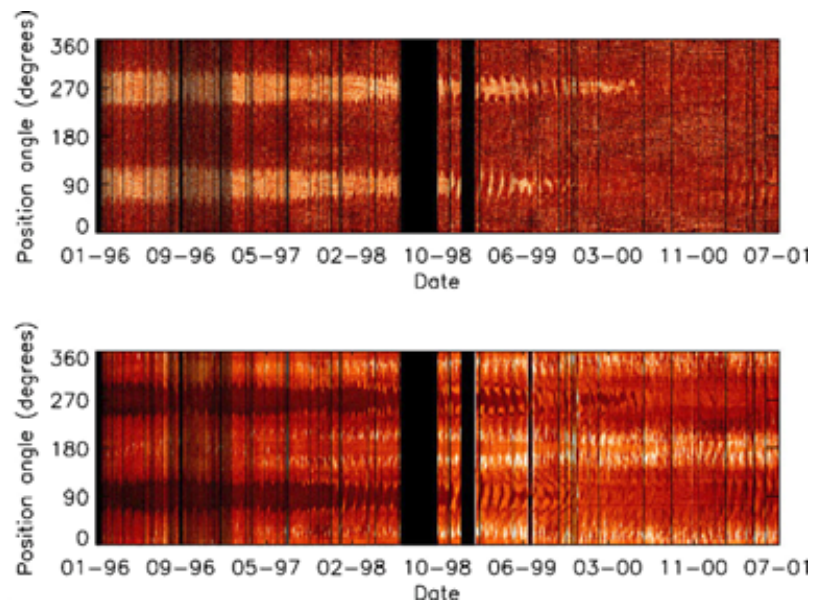

Figure 3. Synoptic view of the time variations of the position of the chromospheric limbs (at the top) and the corresponding intensities (at the bottom) measured with EIT/SoHO images in the HeII line (from F. Auchére).

\section{Discussion and Conclusions}

When the chromospheric prolateness, which is well apparent at solar minimum, was evaluated, the first impression was that it reflects the influence of the global dipolar field of the Sun. Indeed, Roberts, 1944 already used deep H $\alpha$ filtergrams to demonstrate that the distribution of spikes seen around the occulting disk of his coronagraph corresponds to what is expected from a magnetic dipole seeded well inside the Sun. Much later, these spikes were compared to macro-spicules which are seen using TR emission lines, see e.g. Koutchmy and Loucif, 1991 and Yamauchi et al. 2004, where they appear related to CHs. Not only many spicules erupt permanently, but also small prominence-like eruptions are 
seen. These eruptions appear to be recurrent, a property which has been noticed before watching spikes, see Koutchmy and Loucif, 1991.

Simultaneous observations were taken above a polar region in $\mathrm{H} \alpha$ at ground-based and in HeII 304 line from space (EIT/SoHO) by Georgakilas and Koutchmy, 1998. The time sequence illustrates the difference seen between the behaviour of the dynamical chromosphere observed simultaneously in a cool line ( $\mathrm{H} \alpha)$ and in a TR line (HeII 304). Obvious spikes and macro-spicules can easily be identified without being possible to claim any definite correlation, a situation already described by Mariska, 1992 when discussing the TR physics to demonstrate that $1 \mathrm{D}$ models are not adequate. Recently Alissandrakis (private communication) re-examined the question on the light of Ly $\alpha$ TRACE observations, with yet rather different results.

Now, SXR jets and jetlets are also observed in polar regions at sunspot minimum, see Koutchmy et al. 1998, telling us that fast and hot events are present in the corona, exactly what is needed to drive the fast solar wind. In addition, we note the similarity between the azimutal distribution of the fast wind as recorded from Ulysses and the prolateness effect. An obvious similarity also exists regarding the solar cycle variations. R. Woo and Sh. Habbal (see e.g. Woo et al. 2000) promoted for a long time the idea that it is important not to limit the source of the fast wind to the CHs areas, but to also involve the quiet Sun. The prolateness effect we measured seems to bring one more argument in favour of this view. To go further to understand the process of emergence of the small scale magnetic field generated in lower layers, the role of the global fields and the production of small scale dynamical events, capable to heat the high corona and lift a large part of the outer chromosphere, we clearly need more observations. At least the measurements of the prolateness effect is feasible, even at ground-based, and should be made more often to look at a possible longitudinal effect and also get more variabilities to correlate with other parameters, including the $\mathrm{CHs}$ areas.

\section{References}

Auchere, F., Boulade, S., Koutchmy, S., Smartt, R.N., Delaboudiniere, J.P., Georgakilas, A., Gurman, J.B., and Artzner, G.E.: 1998, Astron. Astrophys 366, L57

Dara, H.C., Koutchmy, S., and Suematsu, Y.: 1998, in S. Koutchmy, P. Martens, and K. Shibata (eds.) Solar Jets and Coronal Plumes, Proc. Int. Meeting, Guadeloupe, France, 23-28 February 1998, ESA 421, 255

Dunn, R.B. 1965, Photometry of the Solar Chromosphere, Thesis, Environmental Research Papers AFCL, No 109

Filippov, B. and Koutchmy, S.: 2000 Solar Phys. 196, 311

Fracastoro, M.G.: 1948, Pub. R. Oss. Arcetri 64, 44

Georgakilas, A.A., Koutchmy, S., and Alissandrakis C.E.: 1999, Astron. Astrophys. 341, 610

Huber, M.C.E. Foukal, P.V. Noyes, R.W. Reeves, E.M. Schmahl, E.J. Timothy, J.G. Vernazza, J.E. and Johannesson, A. and Zirin, H.: 1996, Astrophys. J. 471, 510

Koutchmy, S. and Loucif, M. 1991, Mechanisms of Chromospheric and Coronal Heating in Proceedings of the International Conference, Heidelberg, 5-8 June 1990, Ulmschneider, P. Priest, E.R. and Rosner, R. Eds. Springer-Verlag

Koutchmy, S., Smartt, R.N., Auchere, F., Boulade, S.,Gurman, J.B.,Baudin, F., Delaboudiniere, J.P., Georgakilas, A., Hegwer, S., Armstrong, B., Gilliam, D., and Gilliam, L.: 1998, NOAO Newsletter, 56, 7

Koutchmy, S. Hara, H. Shibata, K. Suematsu, Y. and Reardon, K.: 1998, in Observational Plasma Astrophysics: Five Years of Yohkoh and Beyond, T. Watanabe et al. Eds, Kluwer A\&SSL, 229, 87

Mariska, J.T.: 1992, The Solar Transition Region, Cambridge Astrophysics Series, 23

Roberts, W.O.: 1944, Astrophys. J., 101, 136

Rozelot, J.P. and Rosch, J.: 1996, Compt. Rend. Acad. Sci. Paris 322, Serie IIb, 637 
Secchi, S.J.: 1877, in 'Le Soleil', Gauthier-Villars, p. 38

Suematsu, Y.: 1998, in S. Koutchmy, P. Martens, and K. Shibata (eds.) Solar Jets and Coronal Plumes, Proc. Int. Meeting, Guadeloupe, France, 23-28 February 1998, ESA 421, 19

Suematsu, Y., Shibata, K., Nishikawa, T., and Kitai, R.: 1982, Solar Phys. 75, 99

Vernazza, J.E., Avrett, E.H., and Loeser, R.: 1981, Astrophys. J. Supplement 45, 2108

Woo, R. Habbal, S.R. Howard, R.A. and Korendyke, C.M.: 1999, Astrophys. J. 513, 961

Vilinga, J. and Koutchmy, S. 2005, ASP Conf. Series 346, 269

Zachariadis, Th. G., Georgakilas, A.A., Koutchmy, S., and Alissandrakis, C.E.: 1999, Solar Phys. 184,77

Zhang, J., White, S.M., and Kundu, M.R.: 1998, Astrophys.J. 504, L127 